\title{
Resistencia a la corrosión y desgaste de recubrimientos de TiN obtenidos por PVD
}

\author{
A. Conde*, C. Navas*y J.J. de Damborenea* \\ Resumen Los tratamientos de endurecimiento superficial de aleaciones metálicas se utilizan para \\ lograr capas de muy elevada dureza, resistencia a la corrosión y desgaste. En este trabajo, se \\ estudia el comportamiento de recubrimientos de TiN obtenidos mediante PVD sobre aceros \\ de herramientas. Las capas obtenidas se caracterizan mediante microscopía electrónica de \\ barrido, difracción de rayos X y XPS. Se analiza el comportamiento frente a la corrosión de \\ las capas protectoras, así como la influencia del substrato en su comportamiento en \\ soluciones de $\mathrm{NaCl}$. Finalmente, se realizan ensayos de desgaste por deslizamiento en seco \\ mediante la técnica de pin-on-disk, comprobándose la mayor resistencia de estas capas.
}

Palabras clave TiN. XPS. Recubrimientos duros. Corrosión. Desgaste.

\section{Corrosion and wear resistance of PVD-TiN coatings}

\begin{abstract}
Surface hardening techniques for metallic alloys are widely used to achieve layers of very high hardness and corrosion and wear resistance. In the present paper TiN coatings are obtained by PVD on a tool steel. The films are characterized by SEM, X-ray diffraction and XPS. Corrosion behaviour in $\mathrm{NaCl}$ solution showed the importance of the presence of defects on the PVD coating due to the different electrochemical behaviour of the steel base. Pin-on-disk measurements at approximately $40 \% \mathrm{RH}$ against a corundum $\left(\mathrm{Al}_{2} \mathrm{O}_{3}\right)$ ball showed a significant decrease in the wear rate compared with the results for the standard tool steel.
\end{abstract}

Keywords TiN. XPS. Hard coatings. Corrosion. Wear.

\section{INTRODUCCIÓN}

En general, las técnicas de modificación superficial son ampliamente utilizadas para el tratamiento de metales y aleaciones con el fin de obtener capas delgadas de muy elevada dureza, resistencia a la corrosión y desgaste ${ }^{[1 \text { y } 2]}$. De entre ellas, destacan las de deposición en fase vapor (física o química), que permiten obtener recubrimientos ultraduros del tipo $\mathrm{TiN}$ o $\mathrm{CrN}$ que, además de poseer excelentes propiedades químicas y mecánicas son muy apreciados por su carácter decorativo. En este trabajo se caracteriza un recubrimiento de $\mathrm{TiN}$ obtenido mediante la técnica de deposición física en fase vapor (PVD) sobre un acero de rápido de los habitualmente empleados en la fabricación de herramientas y que designaremos por su denominación comercial AISI M2. Estas capas se analizaron me- diante microscopía electrónica de barrido, difracción de rayos X y XPS, y se estudiaron su resistencia al desgaste y a la corrosión.

\section{PROCEDIMIENTO EXPERIMENTAL}

El material base fue un acero rápido de herramientas, AISI M2, con una composición como la que se indica en la tabla I. Los recubrimientos se depositaron sobre la superficie de unos discos de dicho acero previamente pulidos, de $\varnothing=20 \mathrm{~mm}$, y $3 \mathrm{~mm}$ de espesor.

El equipo empleado para la obtención del recubrimiento fue un Tecvac IP35L para deposición por plasma asistido por haz de electrones. De esta forma, se obtuvieron capas de TiN uniformes y homogéneas, aparentemente libres de defectos, de unas $2 \mu \mathrm{m}$ de espesor aproximadamente.

${ }^{*}$ ) Centro Nacional de Investigaciones Metalúrgicas CENIM-CSIC. Av. Gregorio del Amo 8, E-28040 Madird. E-mail: a.conde@cenim.csic.es 
Resistencia a la corrosión y desgaste de recubrimientos de TiN obtenidos por PVD

A. CONDE, C. NAVAS Y J.J. DE DAMBORENEA

Tabla I. Composición química (\% masa) del substrato AISI M2

Table I. Chemical composition (\% wt) of the base AISI M2 steel

\begin{tabular}{lccccccccccc}
\hline \% masa & C & Si & Mn & Cr & P & S & Ni & W & V & Mo & Fe \\
\hline AISI M2 & 0,97 & 0,2 & 0,2 & 4,11 & - & - & - & 6,3 & 1,8 & 4,9 & Resto
\end{tabular}

El análisis de la composición elemental del recubrimiento obtenido se realizó mediante difracción de rayos X y mediante espectroscopía fotoelectrónica de rayos X (XPS). Los espectros de XPS se adquirieron mediante un analizador VG-CLAM de alto vacío con una fuente de radiación $\mathrm{MgK}_{\alpha}$, en el que las muestras y el analizador formaban un ángulo de $90^{\circ}$ (condiciones de operación $5 \mathrm{KeV}$ y corriente de $1,2 \mu \mathrm{A}$ ). Las diferentes contribuciones al enlace de los elementos detectados se ajustaron en los espectros de alta resolución del $\mathrm{O} 1 \mathrm{~s}, \mathrm{Ti}$ $2 \mathrm{p}, \mathrm{N} 1 \mathrm{~s}$ y C $1 \mathrm{~s}$. Las energías de enlace de los picos se corrigieron respecto a la del pico del C 1 s a $285,0 \mathrm{eV}$. Se obtuvo información de la composición de las diferentes muestras a distintos tiempos de bombardeo iónico con gas argón.

En el estudio del comportamiento frente a la corrosión se realizaron curvas potenciodinámicas en $\mathrm{NaCl}$ 0,6 M. Estos ensayos se llevaron a cabo en una célula de corrosión convencional con tres electrodos. La superficie de la muestra, de $0,5 \mathrm{~cm}^{2}$, actuó como electrodo de trabajo. Se utilizó un hilo de platino como contraelectrodo para asegurar una distribución homogénea de la corriente. Finalmente, los potenciales se refirieron a un electrodo de calomelanos saturado (ECS) estándar. Las muestras se sumergieron en la solución correspondiente y, una vez estabilizado el potencial de corrosión, se les aplicó un barrido desde $-500 \mathrm{mV} / \mathrm{ECS}$ con respecto al potencial de corrosión en la solución de $\mathrm{NaCl}$, cortándose al alcanzar una densidad de corriente superior a $10^{-4} \mathrm{~mA} / \mathrm{cm}^{2}$.

La resistencia al desgaste se evaluó mediante el sistema de "bola sobre disco" (pin on disk, según la terminología aceptada en la literatura). El par tribológico acoplado estaba constituido por la muestra -disco que gira bajo la bola- y una bola de alúmina de $\varnothing=3 \mathrm{~mm}$. Los ensayos se realizaron a velocidad de deslizamiento constante, $0,1 \mathrm{~m} / \mathrm{s}$, con una carga aplicada de $0,5 \mathrm{~N}$ a temperatura ambiente y HR del $40 \%$. Los radios del surco de desgaste eran de $2 \mathrm{~mm}$, aproximadamente, y fueron analizados mediante microscopía óptica de barrido
(SEM) y análisis de dispersión de energías (EDS). La distancia deslizada fue desde 250 a $1000 \mathrm{~m}$. Los ensayos de desgaste se realizaron conforme a la norma ASTM G99-95a, así como la evaluación de la pérdida de volumen de los diferentes materiales.

\section{RESULTADOS Y DISCUSIÓN}

El espectro general de la superficie realizado mediante XPS obtenido a diferentes tiempos de sputtering indicaba la presencia de $\mathrm{O} 1 \mathrm{~s}, \mathrm{Ti} 2 \mathrm{p}, \mathrm{N} 1 \mathrm{~s}$ y C 1 s.

La deconvolución de la ventana de alta resolución del N 1s, figura 1, indica que el pico del nitrógeno está constituido, en realidad, por dos contribuciones. La primera, de mayor intensidad, se localiza en una energía de enlace de 396,7 eV que se corresponde con el $\mathrm{TiN}$, seguida de un ligero aumento de la intensidad electrónica en energías del orden de, 398,1 eV que podría estar relacionada con la presencia de un enlace $\mathrm{N}-\mathrm{O}$ en forma de oxinitruro de titanio. De ser este su origen, su presencia debería revelarse también en la ventana de alta resolución del titanio mediante un ligero ahombramiento del espectro en valores de B.E. próximas a 457,0 eV.

Efectivamente, el análisis de la ventana del $\mathrm{Ti}$ $2 p$, figura 2 , muestra que junto a los dos picos del titanio correspondientes al estado $2 \mathrm{p}_{3 / 2}$ y $2 \mathrm{p}_{1 / 2}$ del TiN localizados en 455,0 y 461,0 eV, respectivamente, aparece también un pequeño hombro - o aumento de intensidad- en energías del orden de $456,3 \mathrm{eV}$ que, en principio, corroboraría la presencia del oxinituro antes mencionados y que está de acuerdo con lo observado por otros autores ${ }^{[3 \text { y } 4]}$.

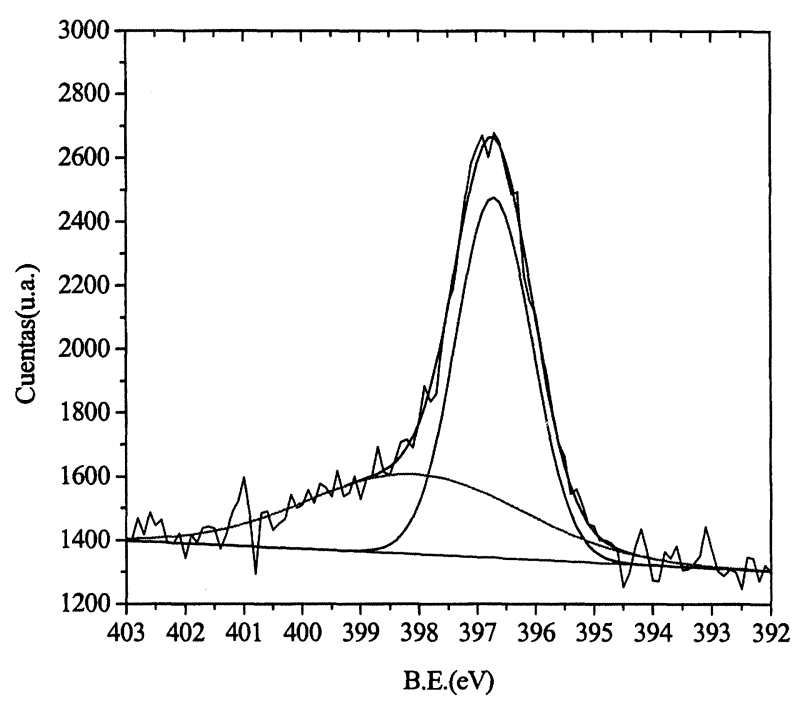

Figura 1. Ventana de alta resolución del $\mathrm{N} 1 \mathrm{~s}$.

Figure 1. N 1s photoelectron spectra.

Rev. Metal. Madrid Vol. Extr. (2005) 457-462 


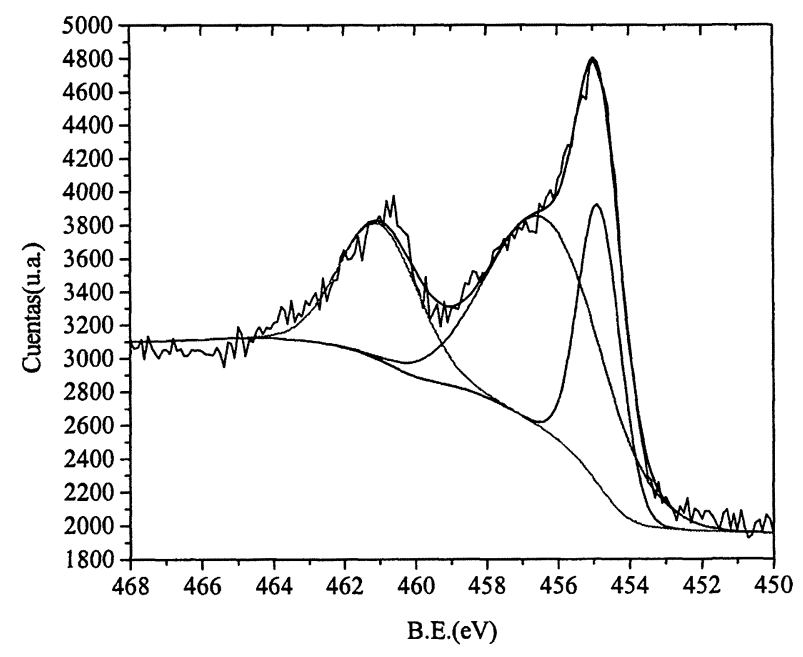

Figura 2. Ventana de alta resolución del Ti 2p.

Figure 2. High resolution XPS Ti $2 p$ spectra of the TiN film.

Finalmente, el pico del oxígeno, figura 3, revela cierta asimetría. Tras la deconvolución y los ajustes realizados con funciones gaussianas, se pone de manifiesto que el pico del oxígeno está constituido, en realidad, por dos picos centrados en energías de enlace de 530,3 y 532,4 eV. El primero, localizado, en las energías más bajas, indica la existencia de óxidos sobre la superficie de la muestra, concretamente $\mathrm{TiO}_{2}$, mientras que el segundo pico estaría relacionado con otro tipo de óxidos o subóxidos de estequiometría diferente.

Por tanto, este análisis de la superficie de las muestras depositadas por PVD sobre AISI M2 indicó que la parte mas externa del recubrimiento de

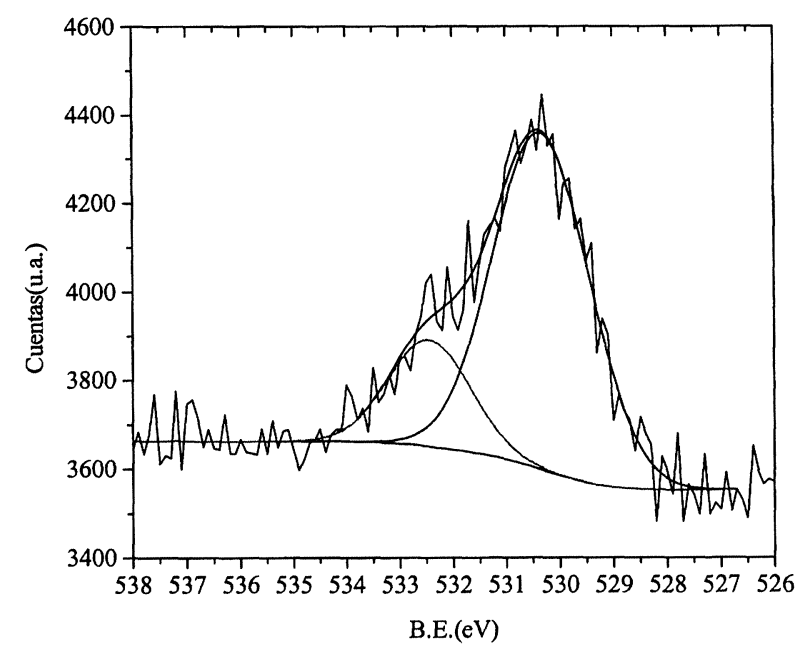

Figura 3. Ventana del $\mathrm{O} 1 \mathrm{~s}$.

Figure 3. O is spectrum of TiN sample.
TiN está fundamentalmente compuesta de TiN. Junto a él, es posible encontrar, en menor proporción, oxinitruros y óxidos de titanio, resultado de la reacción del nitruro metálico con la atmósfera que le rodea.

Para un análisis más preciso del grueso del recubrimiento se utilizó la técnica de análisis de difracción de rayos X. Los resultados confirmaron la presencia de un sólo tipo de nitruros en la capa depositada. Su estequiometría es la del $\mathrm{TiN}$ y posee, además, una marcada textura, tal y como se deduce de la elevada intensidad del pico del TiN(111) localizado en un valor de $2 \theta=36,37$, figura 4 . Este hecho indica que los nitruros se han formado siguiendo una dirección preferencial, la del plano (111), propia de un crecimiento columnar.

Por otra parte, en dicho espectro aparecen también otros picos correspondientes al hierro. Este resultado apunta dos hechos importantes; por un lado, la existencia de zonas de la muestra que no están recubiertas y, por otro, pone de manifiesto el pequeño espesor de la capa depositada, ya que estos picos correspondientes al substrato desparecen con el aumento de espesor de la capa ${ }^{[5]}$.

Es, precisamente, este espesor tan bajo y la posibilidad de que existan poros o defectos en la capa lo que van a condicionar las propiedades protectoras de los recubrimientos de PVD. Esta situación es especialmente perniciosa, desde un punto de vista electroquímico, en este tipo de sistemas debido a que, en la mayoría de los casos, estos recubrimientos son mucho más nobles que el substrato sobre el que se depositan. De manera que cuando el material se expone a un medio agresivo, éste penetra a

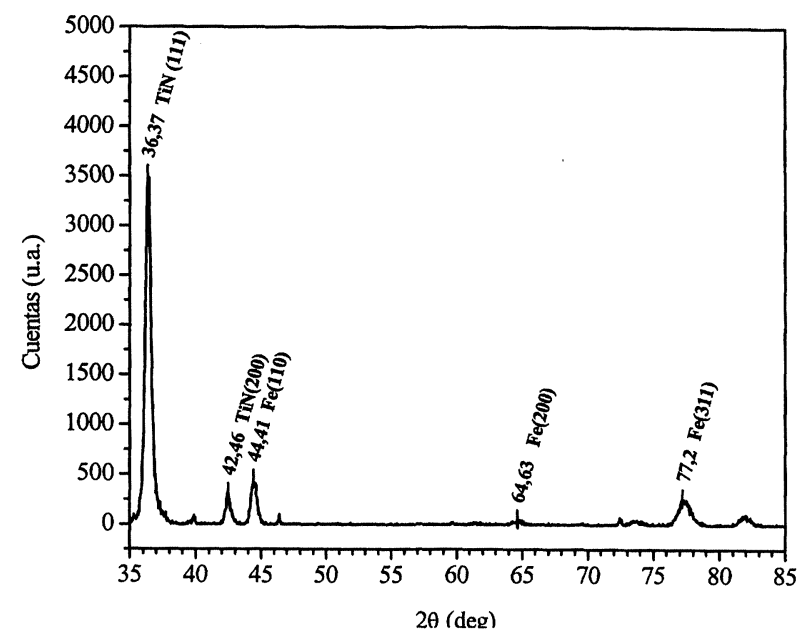

Figura 4. Espectro de difracción de rayos $X$.

Figure 4. X-ray diffraction patterns for TiN layer. 
través de los defectos, originando la aparición de un par galvánico entre el substrato y el recubrimiento. Esta situación, normalmente, conduce a un severo ataque del substrato metálico, superior, incluso, al que experimentaría dicho substrato sin recubrir. Es éste, precisamente, el caso que se tiene en estos recubrimientos de TiN sobre AISI M2 de bajo espesor. En la figura 5, se recogen dos curvas de polarización realizadas sobre zonas distintas de la superficie. En principio, sorprende la total ausencia de pasividad en la rama anódica esperable
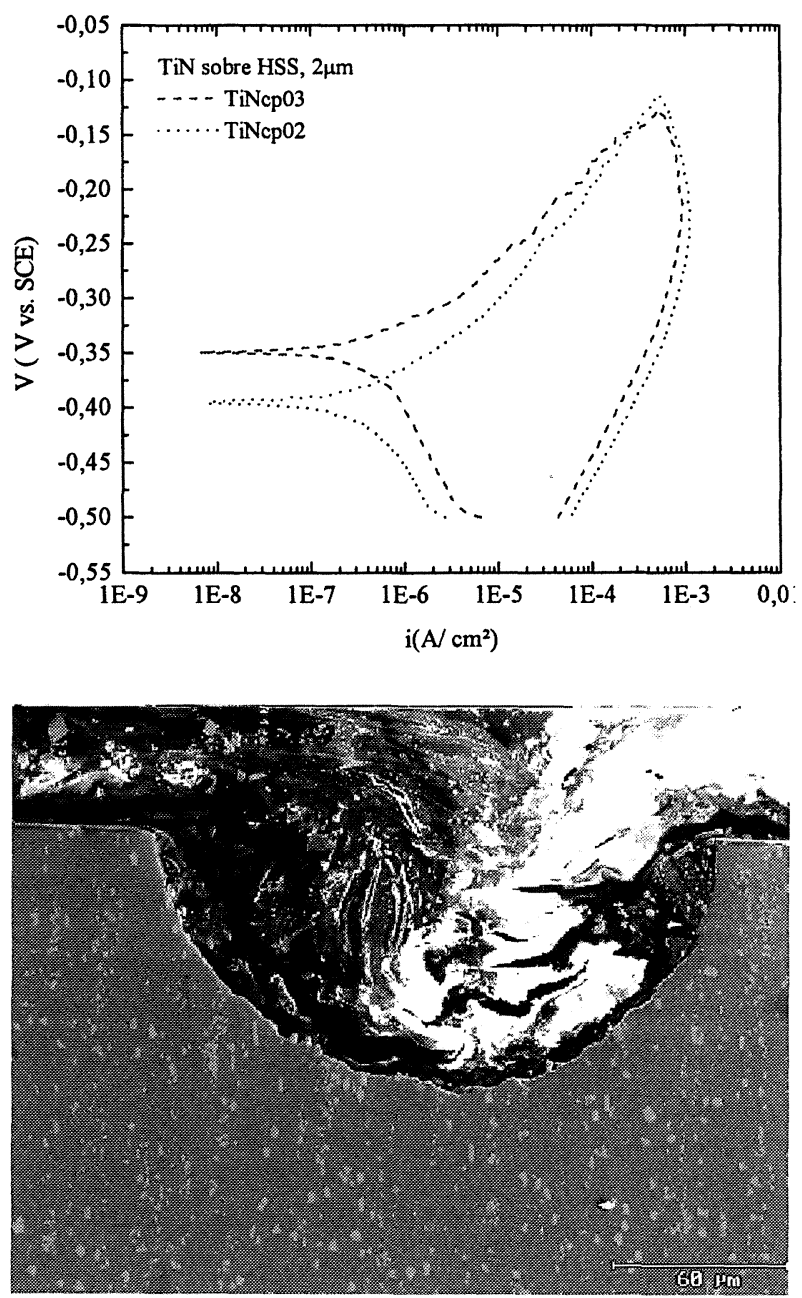

Figura 5. a) Curva de polarización del TiN depositado sobre AISI M2. Tanto el valor de $E_{\text {corr }}$ más anódico como la amplia histéresis de las curvas revelan la influencia del substrato en la respuesta del sistema. b) Corte transversal de la muestra ensayada tras la realización de la curva de polarización.

Figure 5. a) Polarisation curve of TiN on AISI M2. The Ecorr value and the hysteresis in the reverse scans indicates the influence of the base steel. The corrosion potential $\left(E_{\text {corr }}\right)$ of the TiN should be higher and the corrosion current density ( $i_{\text {corr }}$ l lower than the observed. b) Cross-section of a tested sampleafter polarisation curve revealed the formation of pitting. en un recubrimiento de $\mathrm{TiN}$, dada su baja inercia química. Muy al contrario, las curvas revelan un comportamiento muy activo con una histéresis muy acusada en la rama anódica de retorno. Dicha histéresis indica la formación de picaduras que permiten la aparición del par galvánico entre el recubrimiento y el substrato, provocando una rápida disolución del mismo dando lugar a un severo ataque del mismo, figura 5 .

Aunque algunos autores apuntan a que un aumento de espesor de las capas depositadas contribuiría a mejorar la protección frente a la corrosión, otros, afirman que el incremento de espesor contribuye también a un notable aumento de la densidad de defectos y, por tanto, tal mejora respecto a la corrosión no tendría lugar ${ }^{[6]}$. Parece que la presencia, o no, de defectos en las capas depositadas por PVD, está íntimamente ligada al proceso de crecimiento de la capa, es decir, a su estructura. Se ha comprobado que capas de $\mathrm{TiN}$ y $\mathrm{CrN}$ del mismo espesor, obtenidas de forma análoga por PVD, tienen propiedades protectoras distintas. Esto es debido a que los nitruros de cromo crecen con una estructura equiaxial de mayor compacidad respecto de la columnar obtenida para el $\mathrm{TiN}$, haciendo que el camino de difusión del medio al substrato no sea tan directo como en el caso del TiN ${ }^{[7]}$.

Una vez caracterizados, su estructura, composición y comportamiento frente a la corrosión se procedió a evaluar la resistencia al desgaste en seco de las capas de TiN de $2 \mu \mathrm{m}$ de espesor depositadas sobre el substrato AISI M2 mediante ensayos pin-on-disk.

En la figura 6, se recoge la pérdida de volumen en función del número de ciclos deslizados para la capa de TiN y el acero base. Como puede verse, en los primeros 40.000 ciclos $-500 \mathrm{~m}$ deslizados- la pérdida de volumen del AISI M2 es visiblemente superior al del TiN. Conforme el número de ciclos aumenta, la pérdida de material del recubrimiento se aproxima a la del substrato, debido a que el desgaste progresivo de la capa de $\mathrm{TiN}$ va dejando expuesto al material base. A consecuencia de esto, el coeficiente de fricción del recubrimiento también varía durante el ensayo, figura 7. Inicialmente, el TiN posee un elevado coeficiente de fricción de aproximadamente 0,9 , para luego disminuir y alcanzar el valor del substrato AISI M2, una vez que la capa se ha desgastado por completo. Estos resultados permiten estimar la duración total del recubrimiento de TiN en unos 50.000 ciclos $-650 \mathrm{~m}$ para las condiciones ensayadas. Por el contrario, el coeficiente de fricción del acero del susbtrato se 


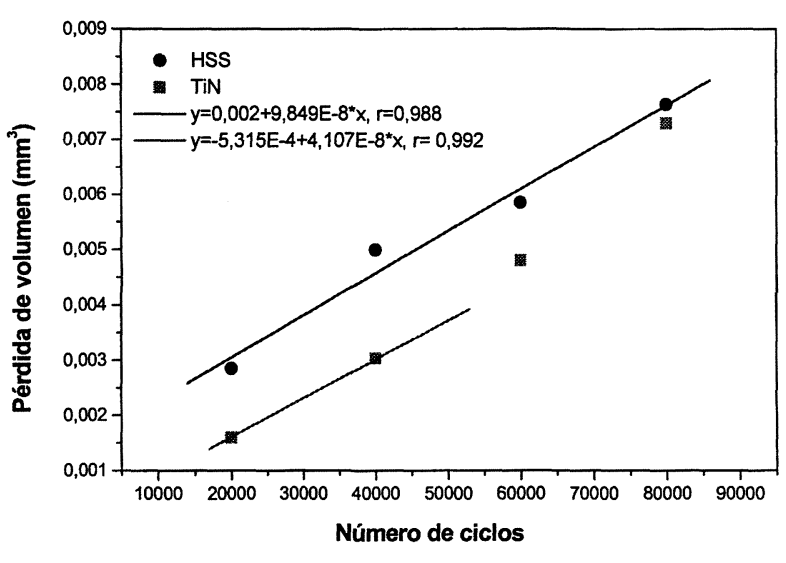

Figura 6. Evolución de la pérdida de volumen en función del número de ciclos deslizados para una velocidad lineal de $0,1 \mathrm{~m} / \mathrm{s}$ y una carga aplicada de $0,5 \mathrm{~N}$.

Figure 6. Evolution of the wear volume as a function of the sliding speed $(0.1 \mathrm{~m} / \mathrm{s})$ and the applied load $(0.5 \mathrm{~N})$.

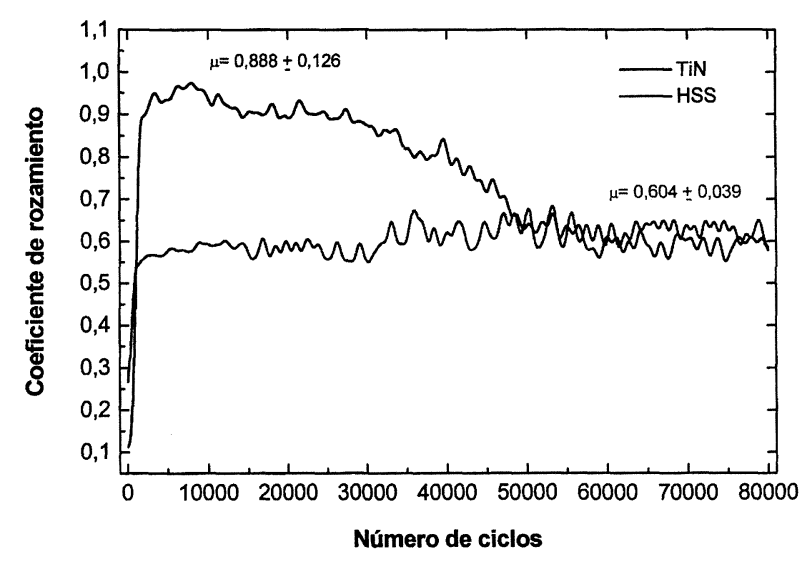

Figura 7. Coeficiente de fricción de los materiales deslizando contra una bola de $\mathrm{Al}_{2} \mathrm{O}_{3}$.

Figure 7. Friction coefficient of the PVD-TiN coating and the steel against the $\mathrm{Al}_{2} \mathrm{O}_{3}$ ball.

mantuvo prácticamente constante durante todo el ensayo. Su menor valor, en comparación con el TiN, indica la formación de una eficaz capa lubricante en la zona de contacto.

La velocidad de desgaste, o volumen perdido por unidad de distancia deslizada, de la capa de TiN es, esencialmente, constante para la duración de la misma ( 50.000 ciclos), ajustándose a la ecuación de una recta, figura 6. Análogamente, el substrato presenta, también, un desgaste lineal para la distancia total deslizada. En ambos casos, se cumple la ley de desgaste por deslizamiento de $\mathrm{Ar}$ chard, a partir de la cual se determina el coeficiente de desgaste dimensional $(\mathrm{k})^{[8]}$. En este caso, los valores obtenidos para el TiN fueron de $6,536 \cdot 10^{-6}$ $\pm 6,094 \cdot 10^{-7} \mathrm{~mm}^{3} / \mathrm{Nm}$ y de $1,567 \cdot 10^{-5} \pm 1,026 \cdot 10^{-6}$ $\mathrm{mm}^{3} / \mathrm{Nm}$ para el AISI M2.

En la figura 8, se presenta la morfología de las huellas de desgaste generadas sobre ambos materiales deslizando contra alúmina. El análisis de EDS del surco de la capa de TiN, indicó la formación de oxinitruro $\mathrm{N}_{\mathrm{x}}-\mathrm{Ti}_{\mathrm{i}} \mathrm{O}_{\mathrm{y}}$ y $\mathrm{TiO}_{2}$, después de deslizar $500 \mathrm{~m}$, lo que permite explicar el mecanismo de desgaste de este tipo de materiales ${ }^{[9]}$. Las partículas duras desprendidas por el desgaste permanecen sobre la superficie actuando como un abrasivo. Aunque la bibliografía afirma que el $\mathrm{TiO}_{2}$ es un eficaz lubricante en capas de $\mathrm{TiO}_{2}$ o en capas de $\mathrm{TiN}$ que se han oxidado completamente a $\mathrm{TiO}_{2}$, en el presente trabajo, el óxido encontrado principalmente sobre la superficie desgastada fue el $\mathrm{N}_{\mathrm{x}}-\mathrm{Ti}-\mathrm{O}_{\mathrm{y}}$, de ahí, el elevado coeficiente de fricción exhibido en los recubrimientos de TiN.

Finalmente, los análisis EDS realizados sobre la superficie del AISI M2 indicaron la presencia de óxido de hierro junto con carburos de wolframio, molibdeno y cromo desprendidos de la matriz del acero durante el desgaste. Estos carburos, al quedar atrapados en la zona de contacto acero/alúmina, ejercen un abrasión muy severa sobre el acero debido a su menor dureza. Además, se produce una oxidación parcial de la superficie del acero durante el deslizamiento, que ejerce un efecto lubricante, disminuyendo el coeficiente de fricción hasta hacerlo inferior al obtenido para las capas de TiN.

\section{CONCLUSIONES}

Los recubrimientos de TiN obtenidos por PVD poseen una estructura de granos columnares que facilita la aparición de defectos. Dada la gran diferencia en el potencial electroquímico entre el recubrimiento y el substrato, la existencia de poros limita las propiedades protectoras del recubrimiento. Desde el punto de vista del desgaste, esta limitación no supone merma de las propiedades, siempre y cuando las piezas estén sometidas a un desgaste en seco, tal y como lo demuestra la menor tasa de desgaste del $\mathrm{TiN}$ respecto del acero base.

\section{REFERENCIAS}

[1] R.L. Martín-Palma, M. Manso, J.M. Martínez Duart, A. Conde and J. de Damborenea. J. Vac. Sci. Technol. A. 21 (2003) 1.635-1.638. 

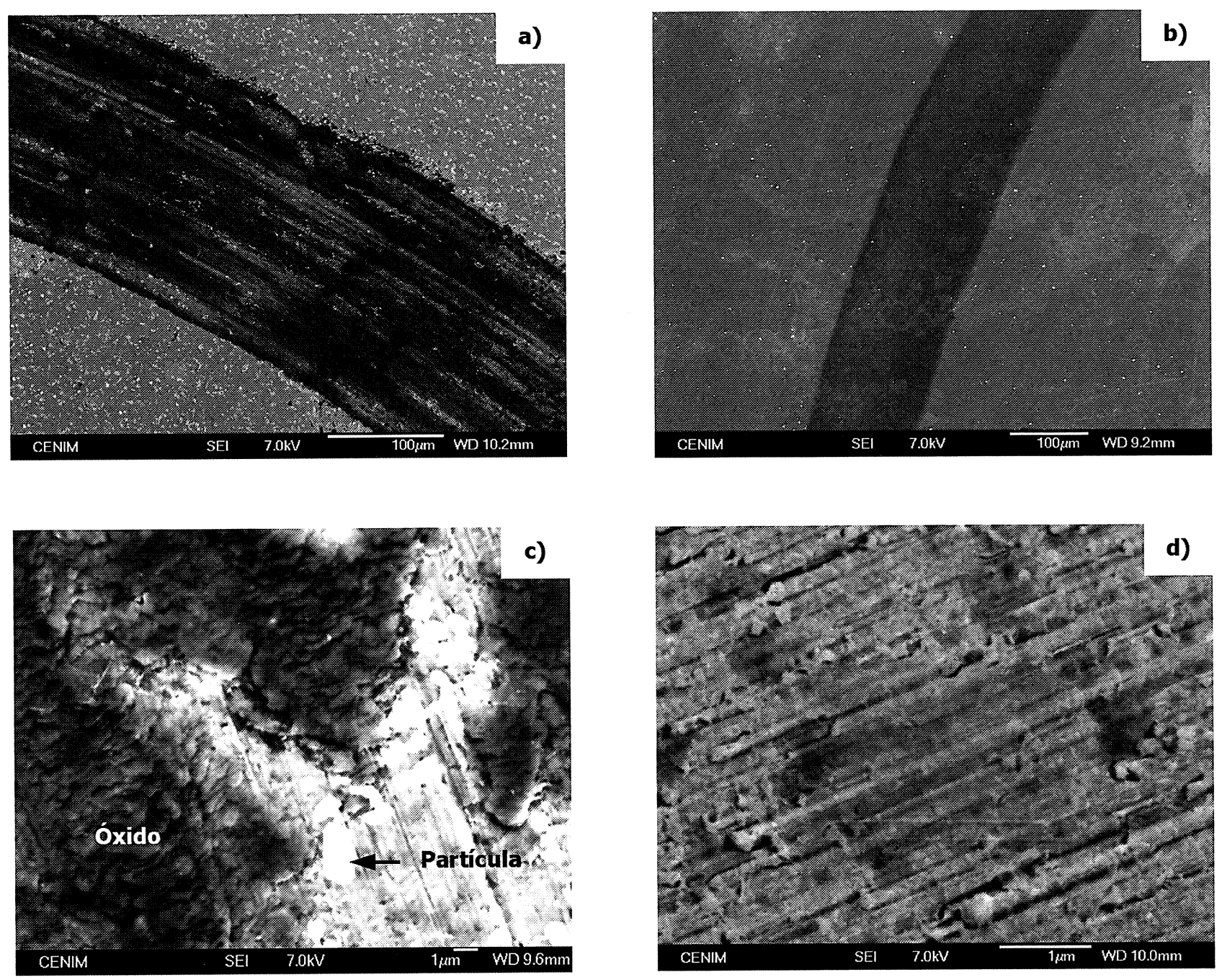

Figura 8. Micrografías de las superficies de desgaste generadas después de deslizar $500 \mathrm{~m}$ contra alúmina para el acero base, a) y c), y para la capa de TiN, b) y d).

Figure 8. SEM micrograph of worn surface after tests at applied load of $0.5 \mathrm{~N}$ and $500 \mathrm{~m}$ of sliding against the $\mathrm{Al}_{2} \mathrm{O}$ for the AISI M2 steel ( $a$ and $c$ ) and the TiN (b and d)

[2] G. Garcés, P. Pérez, P. Adeva. Rev. Metal. Madrid 39 (2003) 17-24

[3] Fu-Hsing Lu, Hong-Ying Chen. Thin Solid Films 355-356 (1999) 374-379.

[4] J.F. Marco, A.C. Agudelo, J.R. Gancedo, D. Hanzel. Surface and Interface Análisis 27 (1999) 71-75.

[5] FengQun Lang, Zhiming Yu. Surface and coatings Technology 145 (2001) 80-87

[6] H.A. Jehn. Surf. Coat. Technol. 125 (2000) 212-217.
[7] C. Liu, Q. Bi, A. Mathews. Corros. Sci. 43 (2001) 1.9531.961.

[8] I.M. Hutchings, Tribology: Friction and Wear of Engineering Materials, Ed. Edward Arnold, A division of Hodder \& Stoughton, 1992, p. 77-132.

[9] Y.M. Zhou, R. Asaki, K. Higashi, W.H. Soe and R. YAMAMOTO. Surf. Coat. Technol. 130 (2000) 9-14. 